\title{
Impact of Imperfect Quality Items on Inventory Management for Two Warehouses with Shortages
}

\author{
Aastha \\ Department of Mathematics and Statistics, \\ Banasthali Vidyapith, Banasthali, 304022, Rajasthan, India. \\ E-mail: aasthapanwar7417@gmail.com \\ Sarla Pareek \\ Department of Mathematics and Statistics, \\ Banasthali Vidyapith, Banasthali, 304022, Rajasthan, India. \\ E-mail: psarla13@gmail.com \\ Leopoldo Eduardo Cárdenas-Barrón \\ Department of Industrial and Systems Engineering, \\ School of Engineering and Sciences, Tecnológico de Monterrey, \\ E. Garza Sada 2501 Sur, C.P. 64849, Monterrey, Nuevo León, México. \\ E-mail: lecarden@tec.mx

\section{Mandeep Mittal} \\ Department of Mathematics, Amity Institute of Applied Sciences, \\ Amity University, Noida, 201313, Uttar Pradesh, India. \\ Corresponding author: mittal_mandeep@yahoo.com
}

(Received May 15, 2019; Accepted May 26, 2020)

\begin{abstract}
Generally, the majority of the inventory models work on the concept that overall units produced must be perfect in terms of quality and that the storage capacity of the warehouse is unlimited. In fact, under realistic conditions, it is not possible to manufacture products with complete perfection. Furthermore, there are always some limits associated with storage capacity of the warehouse. This paper formulates an inventory model that considers the impact of imperfect quality items and shortages. The cost of storage in rented warehouse $(\mathrm{RW})$ is greater than own warehouse $(\mathrm{OW})$ due to fact there are better preservation facilities in RW. This work considers that defective items are completely withdrawn after the inspection process. The purpose of this inventory model is to establish the optimal order quantity and backorder size that maximize the total profit. Some numerical examples are solved, and a sensitivity analysis is included.
\end{abstract}

Keywords- Two warehouse, Imperfect quality items, Shortages.

\section{Introduction}

In the traditional economic order quantity (EOQ) inventory model there are two assumptions: i) the manufactured products are of good quality; and ii) all units are stored in one warehouse only. The perfection of products' quality is not always possible because it is affected straightaway by the reliability of manufacturing process and the handling system used during transportation. Furthermore, the availability of storage space along with the fixed budget to invest upon the raw materials are limited. Due to the restricted capacity of the own warehouse $(\mathrm{OW})$ there is a need of an extra storage with better preserving facilities which has unlimited storage capacity. This extra storage facility is known as rented warehouse (RW). In this direction, some researchers have built 
International Journal of Mathematical, Engineering and Management Sciences

Vol. 5, No. 5, 869-885, 2020

https://doi.org/10.33889/IJMEMS.2020.5.5.067

inventory models by relaxing the two mentioned assumptions.

One inventory model with warehouse was proposed by Hartley (1976). Later, Sarma (1987) generalized Hartley (1976)'s inventory model by including the shipment cost from rented warehouse to own warehouse. Yang (2004) developed a deteriorating inventory model with shortages under inflation in two-warehouse environment. Moreover, Das et al. (2007) formulated a two-warehouse inventory model with supply chain. Hsieh et al. (2008) studied a two warehouses inventory model by optimizing the total cost for deteriorating items. Chung et al. (2009) extended Salameh and Jaber (2000)'s inventory model by applying two warehouse facilities in it. It is important to mention that Salameh and Jaber (2000) postulated an inventory model in which the products in the lot received are of imperfect quality. Wee et al. (2007) presented another variant of the inventory model of Salameh and Jaber (2000) by imposing the assumption that in each cycle of the supply chain the shortages are backlogged and proved that backordering cost is inversely proportional to total profit. Eroglu and Ozdemir (2007) developed another extension of Salameh and Jaber (2000)'s inventory model in which the shortages are allowed. They suggested that there exists a quantity of items with good quality which can covert the existing demand as well as backorders. They concluded that an increment in the proportion of imperfect items in a lot leads to a decrement in total profit per unit time. Table 1 shows some research works related to inventory models.

Table 1. Some research works related to inventory models

\begin{tabular}{|c|c|c|c|c|}
\hline Author(s) & Supply chain & $\begin{array}{c}\text { Defective } \\
\text { items }\end{array}$ & $\begin{array}{c}\text { Two } \\
\text { warehouses }\end{array}$ & Shortage \\
\hline Salameh and Jaber (2000) & $\checkmark$ & $\checkmark$ & & \\
\hline Cárdenas-Barrón (2000) & $\checkmark$ & $\checkmark$ & & \\
\hline Goyal and Cárdenas-Barrón (2002) & $\checkmark$ & $\checkmark$ & & \\
\hline Yang (2004) & $\checkmark$ & & $\checkmark$ & $\checkmark$ \\
\hline Papachristos and Konstantaras (2006) & $\checkmark$ & $\checkmark$ & & $\checkmark$ \\
\hline Eroglu and Ozdemir (2007) & $\checkmark$ & $\checkmark$ & & $\checkmark$ \\
\hline Das et al. (2007) & $\checkmark$ & & $\checkmark$ & \\
\hline Maddah and Jaber (2008) & $\checkmark$ & $\checkmark$ & & \\
\hline Chung et al. (2009) & $\checkmark$ & $\checkmark$ & $\checkmark$ & \\
\hline Sarkar (2012) & & $\checkmark$ & & \\
\hline Jaggi et al. (2013) & & & $\checkmark$ & $\checkmark$ \\
\hline Jaggi et al. (2014) & & & $\checkmark$ & $\checkmark$ \\
\hline Jaggi et al. (2015a) & & & $\checkmark$ & $\checkmark$ \\
\hline Jaggi et al. (2015b) & & $\checkmark$ & $\checkmark$ & \\
\hline Tiwari et al. (2016) & $\checkmark$ & & $\checkmark$ & \\
\hline Jaggi et al. (2017) & $\checkmark$ & $\checkmark$ & $\checkmark$ & \\
\hline Mukhopadhyay and Goswami (2017) & $\checkmark$ & $\checkmark$ & & $\checkmark$ \\
\hline Yadav et al. (2018) & $\checkmark$ & $\checkmark$ & & \\
\hline This paper & $\checkmark$ & $\checkmark$ & $\checkmark$ & $\checkmark$ \\
\hline
\end{tabular}

This paper develops an inventory model for imperfect quality items with shortages in a two warehouses environment. All shortages are fully backordered. The inventory model accounts the impact of imperfect quality items into the two warehouses on total profit. 
International Journal of Mathematical, Engineering and Management Sciences

Vol. 5, No. 5, 869-885, 2020

https://doi.org/10.33889/IJMEMS.2020.5.5.067

\section{Assumptions and Notation}

\subsection{Assumptions}

(i) A known, constant, and continuous demand rate is considered.

(ii) Instantaneous replenishment rate.

(iii) A single product is considered.

(iv) Proportion of imperfect quality items $\rho$ follows a uniform distribution $[\alpha, \beta]$ where $[0 \leq \alpha \leq \beta \leq 1]$.

(v) The shortage are allowed and fully backlogged.

\subsection{Notation}

The inventory model is built utilizing the following notation.

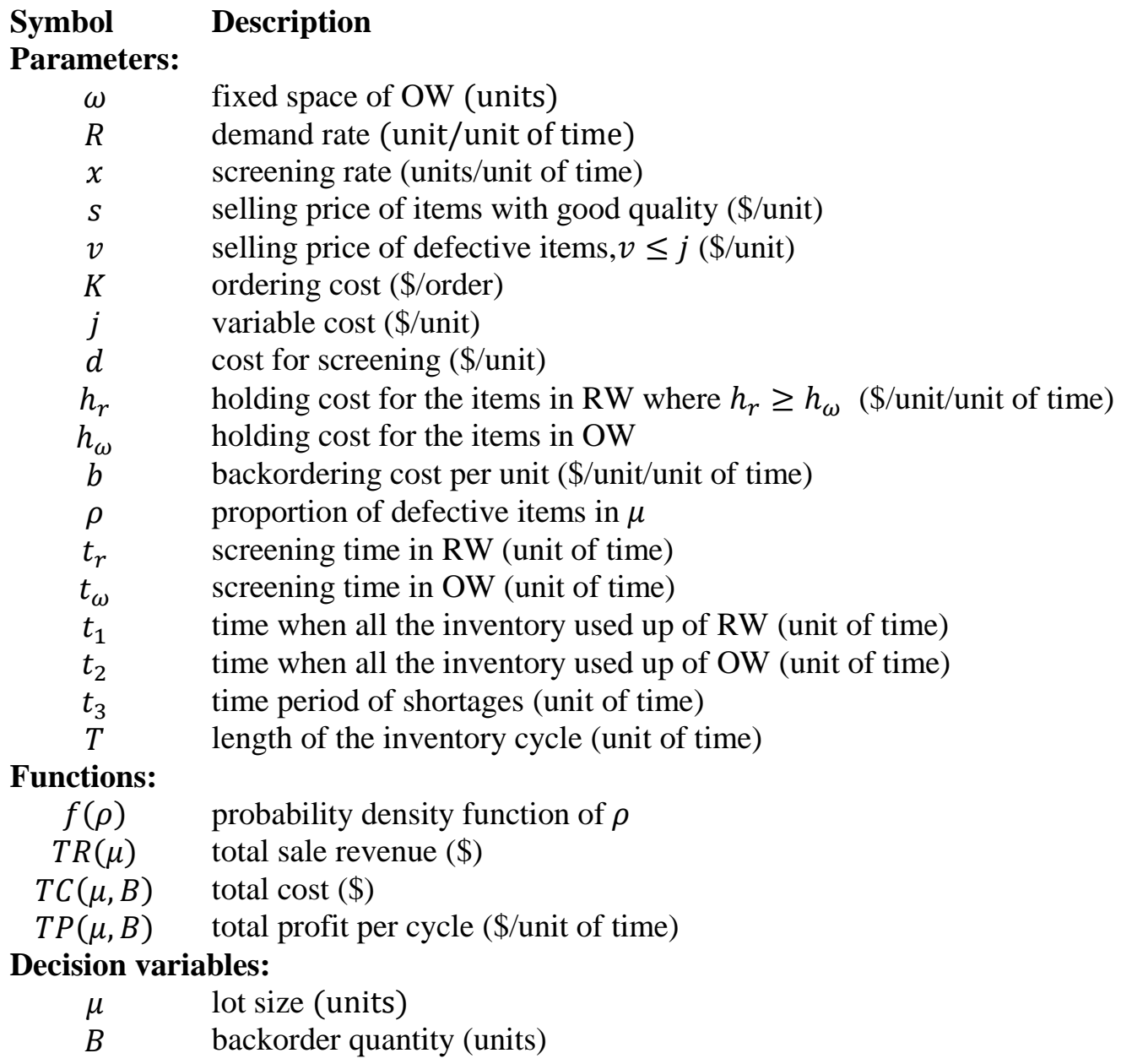

\section{Modelling}

At the beginning a lot size of $\mu$ units come into the inventory system at time $t=0$. It is assumed that from this lot size $\omega$ units are stored in the own warehouse (OW) and $(\mu-\omega-B)$ units are stored in the rented warehouse (RW).The rent warehouse has better preserving facilities than own warehouse which implies that the cost of holding the stock in rent warehouse is higher than that of 
own warehouse. The behavior of the inventory model is illustrated in Figure 1 and Figure 2 for two cases that can occur. Due to certain reasons such as improper transport, low labor skills, low quality of raw material, among others, the production process manufactures some goods that are not of high-quality. Due to this, a screening process must be conducted with the rate of $x$ units per unit time when the complete lot enters the inventory system. It is presumed that each lot $\mu$ contains an $\rho$ percent of imperfect items, where $\rho$ is a random variable whose probability density function is, $f(\rho)$, and its mean is $E[\rho]=\rho$. Thus, the lot $\mu$ has $\rho \mu$ imperfect quality items and $(1-\rho) \mu$ perfect items. The imperfect quality items are kept in store and sold at the termination of the screening phase $\left(t_{r}\right)$ at a salvage value of $v$ per unit, where $v \leq j$. During time $t_{1}$ all the demand is fulfilled from RW until the inventory level of RW reduces to zero. After, the upcoming demand is now covered from $\mathrm{OW}$ during time $t_{2}$. When the inventory level reduces to zero in $\mathrm{OW}$, then the shortages occur during the time $t_{3}$. The time horizon $(T)$ of total inventory is given by

$T=(1-\rho) \frac{\mu}{R}+\frac{B \rho}{R}$

The expected value of time horizon $(T)$ is

$E[T]=(1-\mathrm{E}[\rho]) \frac{\mu}{R}+\frac{B E[\rho]}{R}$

Then, the whole revenue is defined as $T R(\mu)$ and the total cost is denoted as $T C(\mu, B)$. The $T R(\mu)$ is the sum of sales of perfect items and the imperfect items,

$T R(\mu)=s \mu(1-\rho)+v \rho \mu$

$T C(\mu, B)=$ ordering cost + purchase cost + screening cost + holding cost + backordering cost

$T C(\mu, B)=K+j \mu+d \mu+h_{r}\left[\left(\frac{1}{2} t_{1}(\mu-\omega-B)(1-\rho)\right)+t_{r} \rho(\mu-\omega-B)\right]+$

$h_{\omega}\left[\omega \rho t_{\omega}+\omega t_{1}(1-\rho)+\frac{(\omega(1-\rho)) t_{2}}{2}\right]+\frac{B b t_{3}}{2}$

The times $t_{r}, t_{\omega}, t_{1}, t_{2}$, and $t_{3}$ (See Figure 1 ) are given by

$$
\begin{aligned}
& t_{r}=\frac{\mu-\omega-B}{x} \\
& t_{\omega}=\frac{\omega}{x} \\
& t_{1}=\frac{(\mu-\omega-B)(1-\rho)}{R} \\
& t_{2}=\frac{\omega(1-\rho)}{R} \\
& t_{3}=\frac{B}{R}
\end{aligned}
$$

By substituting the times $t_{r}, t_{\omega}, t_{1}, t_{2}$, and $t_{3}$ into equation (4), 
International Journal of Mathematical, Engineering and Management Sciences

Vol. 5, No. 5, 869-885, 2020

https://doi.org/10.33889/IJMEMS.2020.5.5.067

$T C(\mu, B)=K+j \mu+d \mu+h_{r}\left[\frac{1}{2 R}(\mu-\omega-B)^{2}(1-\rho)^{2}+\frac{\rho(\mu-\omega-B)^{2}}{x}\right]+$

$h_{\omega}\left[\frac{\omega(\mu-\omega-B)(1-\rho)^{2}}{R}+\frac{\omega^{2} \rho}{x}+\frac{\omega^{2}(1-\rho)^{2}}{2 R}\right]+\frac{B^{2} b}{2 R}$

The total profit per unit of time, $\operatorname{TPU}(\mu, B)$, is determined by the difference between total revenue per unit of time $\frac{T R(\mu)}{T}$ and total cost per unit of time $\frac{T C(\mu, B)}{T}$. Hence,

$T P U(\mu, B)=\frac{T R(\mu)-T C(\mu, B)}{T}$

Since $\rho$ is a random variable that follows a uniform distribution with known p.d.f. $f(\rho)$ then the expected value of total profit $E[T P U(\mu, B)]$ is

$$
\begin{aligned}
& E[T P U(\mu, B)]=\frac{E[T R(\mu)]-E[T C(\mu, B)]}{E[T]} \\
& E[T P U(\mu, B)]=\frac{R}{(1-\mathrm{E}[\rho]) \mu+B E[\rho]}\left[s \mu(1-\mathrm{E}[\rho])+v \mathrm{E}[\rho] \mu-\left(K+j \mu+d \mu+h_{r}\left[\frac{1}{2 R}(\mu-\omega-\right.\right.\right. \\
& \left.\left.\left.B)^{2}(1-\mathrm{E}[\rho])^{2}+\frac{\mathrm{E}[\rho](\mu-\omega-B)^{2}}{x}\right]+h_{\omega}\left[\frac{\omega(\mu-\omega-B)(1-\mathrm{E}[\rho])^{2}}{R}+\frac{\omega^{2} \mathrm{E}[\rho]}{x}+\frac{\omega^{2}(1-\mathrm{E}[\rho])^{2}}{2 R}\right]+\frac{B^{2} b}{2 R}\right)\right]
\end{aligned}
$$

Case I. When $t_{1} \geq t_{\omega}$, this case is illustrated by Figure 1 .

With respect to Case II the total revenue $T R(\mu)$ is given as in Case I and the total cost $T C(\mu, B)$ is given below

$$
\begin{aligned}
& T C(\mu, B)=K+j \mu+d \mu+h_{r}\left[\left(\frac{1}{2} t_{1}(\mu-\omega-B)(1-\rho)\right)+t_{r} \rho(\mu-\omega-B)\right]+ \\
& h_{\omega}\left[\omega t_{1}+\frac{R\left(t_{\omega}-t_{1}\right)^{2}}{2}+\left(t_{\omega}-t_{1}\right)\left(\omega-\left(t_{\omega}-t_{1}\right) R\right)+\frac{1}{2}\left(t_{1}+t_{2}-t_{\omega}\right)\left(\omega-\left(t_{\omega}-t_{1}\right) R-\rho \omega\right)\right]+ \\
& \frac{B b t_{3}}{2}
\end{aligned}
$$

Since,

$t_{1}=\frac{(\mu-\omega-B)(1-\rho)}{R}, t_{r}=\frac{\mu-\omega-B}{x}, t_{\omega}=\frac{\omega}{x}, t_{2}=\frac{\omega(1-\rho)}{R}, t_{3}=\frac{B}{R}$.

Then equation (14) is expressed as follows:

$$
\begin{aligned}
& T C(\mu, B)=K+j \mu+d \mu+h_{r}\left[\frac{1}{2 R}(\mu-\omega-B)^{2}(1-\rho)^{2}+\frac{\rho(\mu-\omega-B)^{2}}{x}\right]+h_{\omega}\left[\frac{(\mu-\omega-B)(1-\rho) \omega}{R}+\right. \\
& R\left(\frac{\omega}{x}-\frac{(\mu-\omega-B)(1-\rho)}{R}\right)^{2}+\left(\frac{\omega}{x}-\frac{(\mu-\omega-B)(1-\rho)}{R}\right)\left(\omega-R\left(\frac{\omega}{x}-\frac{(\mu-\omega-B)(1-\rho)}{R}\right)\right)+ \\
& \left.\frac{1}{2}\left(\frac{(\mu-\omega-B)(1-\rho)+\omega(1-\rho)}{R}-\frac{\omega}{x}\right)\left(\omega-R\left(\frac{\omega}{x}-\frac{(\mu-\omega-B)(1-\rho)}{R}\right)-\rho \omega\right)\right]+\frac{B^{2} b}{2 R}
\end{aligned}
$$


International Journal of Mathematical, Engineering and Management Sciences

Vol. 5, No. 5, 869-885, 2020

https://doi.org/10.33889/IJMEMS.2020.5.5.067

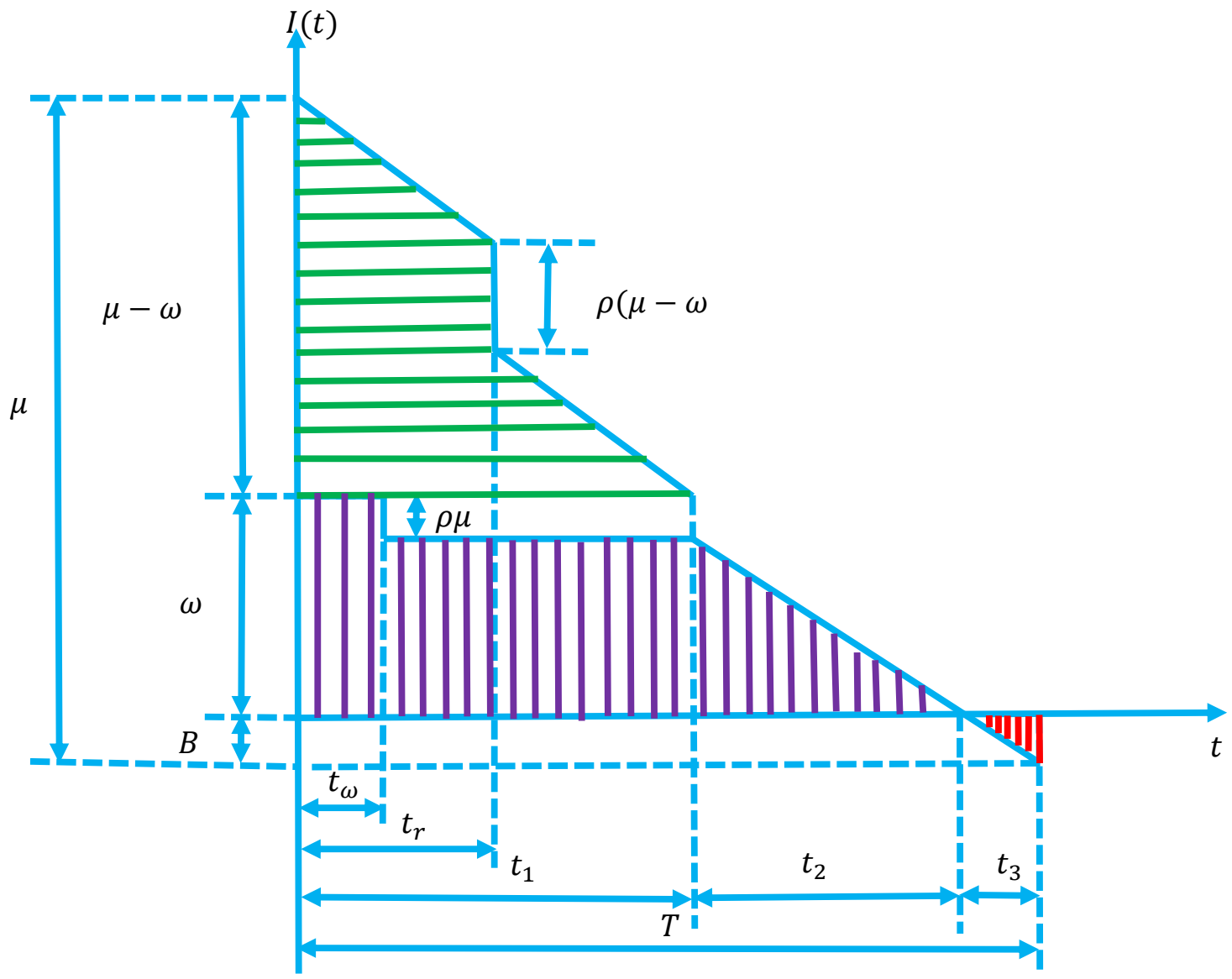

Figure 1 . The inventory level of items in stock of two warehouse if $t_{1} \geq t_{\omega}$

Case II. When $t_{1}<t_{\omega}$, this case is depicted in Figure 2.

The total profit per unit of time, $\operatorname{TPU}(\mu, B)$, is given by

$T P U(\mu, B)=\frac{T R(\mu)-T C(\mu, B)}{T}$

Since $\rho$ is a random variable that follows a uniform distribution with known p.d.f. $f(\rho)$ then the expected value of total profit $E[T P U(B, u)]$ is

$$
\begin{aligned}
& E[T P U(\mu, B)]=\frac{R}{(1-\mathrm{E}[\rho]) \mu+B \mathrm{E}[\rho]}\left[s \mu(1-\mathrm{E}[\rho])+v \mathrm{E}[\rho] \mu-\left(K+j \mu+d \mu+h_{r}\left[\frac{1}{2 R}(\mu-\omega-\right.\right.\right. \\
& \left.B)^{2}(1-\mathrm{E}[\rho])^{2}+\frac{\mathrm{E}[\rho](\mu-\omega-B)^{2}}{x}\right]+h_{\omega}\left[\frac{(\mu-\omega-B)(1-\mathrm{E}[\rho]) \omega}{R}+R\left(\frac{\omega}{x}-\frac{(\mu-\omega-B)(1-\mathrm{E}[\rho])}{R}\right)^{2}+\left(\frac{\omega}{x}-\right.\right. \\
& \left.\frac{(\mu-\omega-B)(1-\mathrm{E}[\rho])}{R}\right)\left(\omega-R\left(\frac{\omega}{x}-\frac{(\mu-\omega-B)(1-\mathrm{E}[\rho])}{R}\right)\right)+\frac{1}{2}\left(\frac{(\mu-\omega-B)(1-\mathrm{E}[\rho])+\omega(1-\mathrm{E}[\rho])}{R}-\frac{\omega}{x}\right)(\omega- \\
& \left.\left.\left.\left.R\left(\frac{\omega}{x}-\frac{(\mu-\omega-B)(1-\mathrm{E}[\rho])}{R}\right)-\mathrm{E}[\rho] \omega\right)\right]+\frac{B^{2} b}{2 R}\right)\right]
\end{aligned}
$$


International Journal of Mathematical, Engineering and Management Sciences

Vol. 5, No. 5, 869-885, 2020

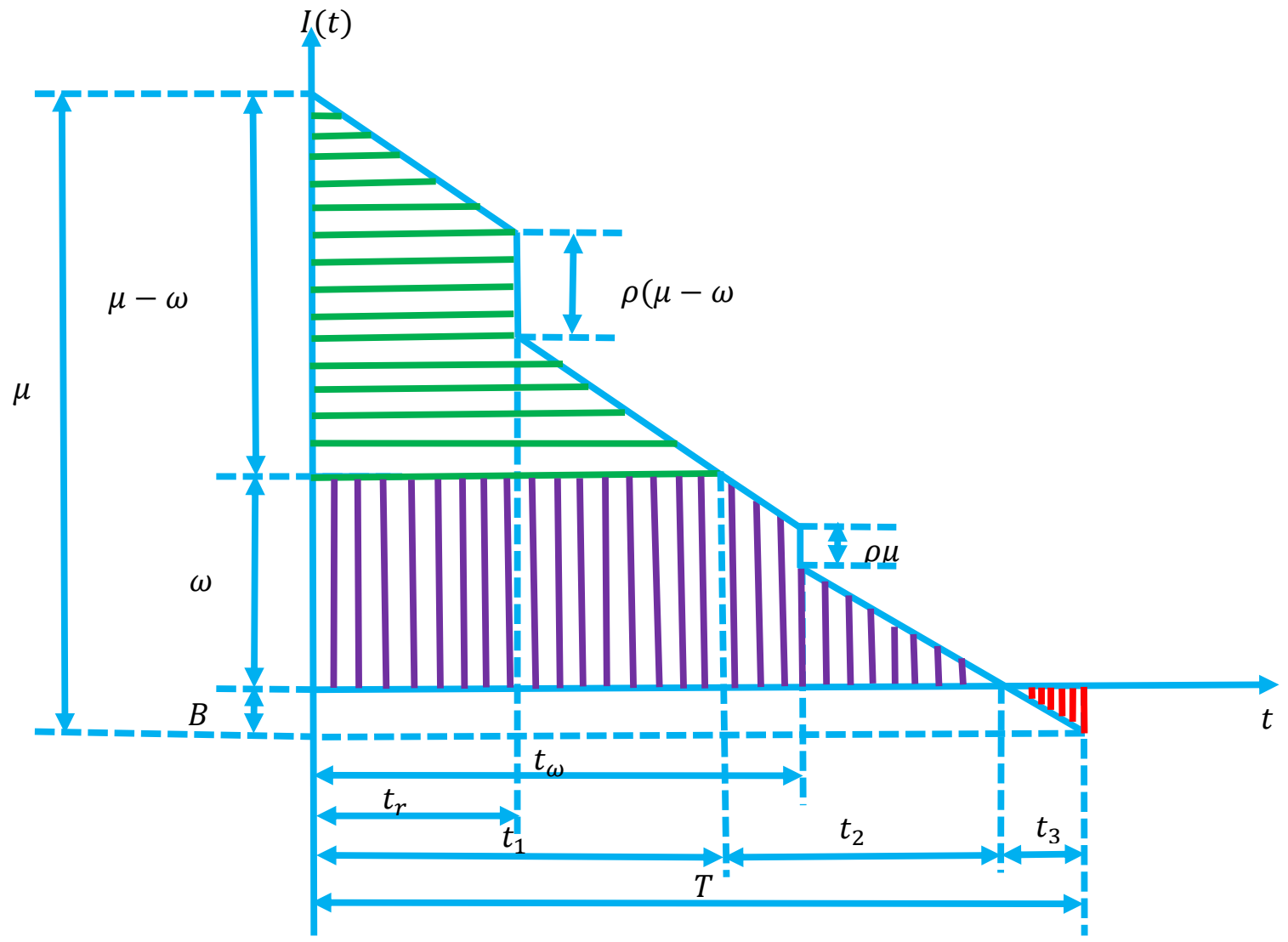

Figure 2. The inventory level of items in stock of two warehouse if $t_{1}<t_{\omega}$

\section{Solution Procedure}

The aim is to calculate the optimal order quantity $\mu^{*}$ and optimal backorder quantity $B^{*}$. The necessary conditions for that the expected profit to be maximized are: $\frac{\partial E[T P U(\mu, B)]}{\partial \mu}=0$ and $\frac{\partial E[T P U(\mu, B)]}{\partial B}=0$. Additionally, for that the expected total profit $E[T P U(\mu, B)]$ to be concave, the sufficient conditions are as follows:

$\left(\frac{\partial^{2} E[T P U(\mu, B)]}{\partial \mu^{2}}\right) \leq 0$

$\left(\frac{\partial^{2} E[T P U(\mu, B)]}{\partial B^{2}}\right) \leq 0$

$\left(\frac{\partial^{2} E[T P U(\mu, B)]}{\partial \mu^{2}}\right)\left(\frac{\partial^{2} E[T P U(\mu, B)]}{\partial B^{2}}\right)-\left(\frac{\partial^{2} E[T P U(\mu, B)]}{\partial \mu \partial B}\right)^{2} \geq 0$

If the solution $\left(\mu^{*}, B^{*}\right)$ satisfies the conditions given by the equations (18), (19) and (20) prove that the function $E[T P U(\mu, B)]$ is strictly concave with a negative-definite Hessian matrix. Therefore, the solution $\left(\mu^{*}, B^{*}\right)$ is optimal. Thus, the solution procedure for each case is presented below. 
International Journal of Mathematical, Engineering and Management Sciences

Vol. 5, No. 5, 869-885, 2020

https://doi.org/10.33889/IJMEMS.2020.5.5.067

Algorithm for Case I. When $t_{1} \geq t_{\omega}$

Step 1. Determine $\mu^{*}$ and $B^{*}$ by solving simultaneously equation (A1) and equation (A2) given in Appendix A.

Step 2. If $\left(\frac{\partial^{2} E[T P U(\mu, B)]}{\partial \mu^{2}}\right) \leq 0$ given in equation (A3); $\left(\frac{\partial^{2} E[T P U(\mu, B)]}{\partial B^{2}}\right) \leq 0$ given in equation (A4) and the Hessian determinant (A3)(A4)-(A5) $\geq 0$ then the solution is optimal.

Step 3. Calculate the expected total profit $E[\operatorname{TPU}(\mu, B)]$ with equation (13).

Step 4. Compute the values of $t_{r}{ }^{*}, t_{w}{ }^{*}, t_{1}{ }^{*}, t_{2}{ }^{*}, t_{3}{ }^{*}$, and $T^{*}$.

Step 5. Report the optimal solution.

Algorithm for Case II. When $t_{1}<t_{\omega}$

Step 1. Calculate $\mu^{*}$ and $B^{*}$ by solving simultaneously equation (A6) and equation (A7) given in Appendix B.

Step 2. If $\left(\frac{\partial^{2} E[T P U(\mu, B)]}{\partial \mu^{2}}\right) \leq 0$ given in equation (A8); $\left(\frac{\partial^{2} E[T P U(\mu, B)]}{\partial B^{2}}\right) \leq 0$ given in equation (A9) and the Hessian determinant (A8)(A9)-(A10) $\geq 0$ then the solution is optimal.

Step 3. Compute the expected total profit $E[T P U(\mu, B)]$ with equation (17).

Step 4. Determine the values of $t_{r}{ }^{*}, t_{w}{ }^{*}, t_{1}{ }^{*}, t_{2}{ }^{*}, t_{3}{ }^{*}$, and $T^{*}$.

Step 5. Report the optimal solution.

\section{Numerical Examples}

This section presents two numerical examples for illustrating the solution of two cases that can happen in the inventory model.

Example 1. This example illustrates the Case I when $t_{1} \geq t_{\omega}$. The values for the parameters are taken from Chung et al. (2009). These values are as follows:

$\omega=800$ units; $R=50000$ units/year; $x=175200$ units/year; $s=50 \$ /$ unit; $v=20$ /unit; $K=100$ $\$$ /order; $j=20 \$$ unit; $d=0.5 \$$ unit; $h_{r}=7 \$$ unit/year; $h_{w}=\$ 5 /$ unit/year. Additionally, the backordering cost is included as $b=10 \$ /$ unit/year;

The proportion of imperfect items is a random variable $(\rho)$ which follows a uniform distribution with p.d.f. as $f(\rho)=\left\{\begin{array}{c}25,0 \leq \rho \leq 0.04 \\ 0, \text { otherwise }\end{array}\right.$. Therefore, $E[\rho]=0.02$.

By applying the algorithm for Case I we obtain the following optimal solution:

$\begin{array}{ccccccccc}\mu^{*} & B^{*} & E\left[T P U\left(\mu^{*}, \mu^{*}\right)\right] & t_{r}{ }^{*} & t_{w}{ }^{*} & t_{1}{ }^{*} & t_{2}{ }^{*} & t_{3}{ }^{*} & T^{*} \\ 8559.474 & 3511.078 & 1444999 & 0.02424884 & 0.00456621 & 0.08326857 & 0.01568 & 0.07022155 & 0.1691701\end{array}$


International Journal of Mathematical, Engineering and Management Sciences

Vol. 5, No. 5, 869-885, 2020

https://doi.org/10.33889/IJMEMS.2020.5.5.067

Notice that $t_{1} \geq t_{\omega}$ as it is expected.

The optimality test that ensures that the solution is optimal is as follows:

$$
\begin{aligned}
& \left(\frac{\partial^{2} E[T P U(\mu, B)]}{\partial \mu^{2}}\right)=-0.07987011 \leq 0 ;\left(\frac{\partial^{2} E[T P U(\mu, B)]}{\partial B^{2}}\right)=-0.001969006 \leq 0 \text { and } \\
& \left(\frac{\partial^{2} E[T P U(\mu, B)]}{\partial \mu^{2}}\right)\left(\frac{\partial^{2} E[T P U(\mu, B)]}{\partial B^{2}}\right)-\left(\frac{\partial^{2} E[T P U(\mu, B)]}{\partial \mu \partial B}\right)^{2}=(-0.07987011)(-0.001969006)- \\
& (0.001233618)^{2}=0.0001557429 \geq 0 .
\end{aligned}
$$

The developed inventory model is realistic in nature as occurrence of shortages is common in the majority of business. This numerical example proves the fact as the proposed inventory model is profitable as compared to Chung et al. (2009)'s inventory model because there is an increment in the total profit of 232971 (1444999-1212028).

Example 2. This example illustrates the Case II when $t_{1}<t_{\omega}$. The data for the parameters are: $\omega=17000$ units; $R=98000$ units/year; $x=175200$ units/year; $s=65$ \$/unit; $v=21$ \$/unit; $K=110 \$ /$ order; $j=26 \$$ unit; $d=0.69 \$ /$ unit; $h_{r}=11$ \$/unit/year; $h_{w}=\$ 7 /$ unit/year; $b=69$ $\$$ /unit/year; $E[\rho]=0.02$.

By applying the algorithm for Case II we obtain the following optimal solution:

$\begin{array}{cclcccccc}\mu^{*} & B^{*} & E\left[\operatorname{TPU}\left(\mu^{*}, \mu^{*}\right)\right] & t_{r}{ }^{*} & t_{w}{ }^{*} & t_{1}{ }^{*} & t_{2}{ }^{*} & t_{3}{ }^{*} & T^{*} \\ 18641.55 & 79.65707 & 3665681 & 0.008914928 & 0.09703196 & 0.01561895 & 0.17 & 0.0008128273 & 0.1864318\end{array}$

Note that $t_{1}<t_{\omega}$ as it is expected.

The optimality test that guarantees that the solution is optimal is given below.

$$
\begin{aligned}
& \left(\frac{\partial^{2} E[T P U(\mu, B)]}{\partial \mu^{2}}\right)=-2.802276 \leq 0 ;\left(\frac{\partial^{2} E[T P U(\mu, B)]}{\partial B^{2}}\right)=-0.004736280 \leq 0 \text { and } \\
& \left(\frac{\partial^{2} E[T P U(\mu, B)]}{\partial \mu^{2}}\right)\left(\frac{\partial^{2} E[T P U(\mu, B)]}{\partial B^{2}}\right)-\left(\frac{\partial^{2} E[T P U(\mu, B)]}{\partial \mu \partial B}\right)^{2}=(-2.802276)(-0.004736280)- \\
& (0.0009596589)^{2}=0.01327144 \geq 0 .
\end{aligned}
$$

\section{Sensitivity Analysis}

The sensitivity analysis is executed for the example 1 . The effects of fraction defective items $(E[\rho])$ on the values of order quantity $\left(\mu^{*}\right)$, backorder quantity $\left(B^{*}\right)$ and the expected total profit $\left(E\left[T P U\left(\mu^{*}, \mu^{*}\right)\right]\right)$ are shown in Figure 3, Figure 4 and Figure 5, respectively. 
International Journal of Mathematical, Engineering and Management Sciences

Vol. 5, No. 5, 869-885, 2020

https://doi.org/10.33889/IJMEMS.2020.5.5.067

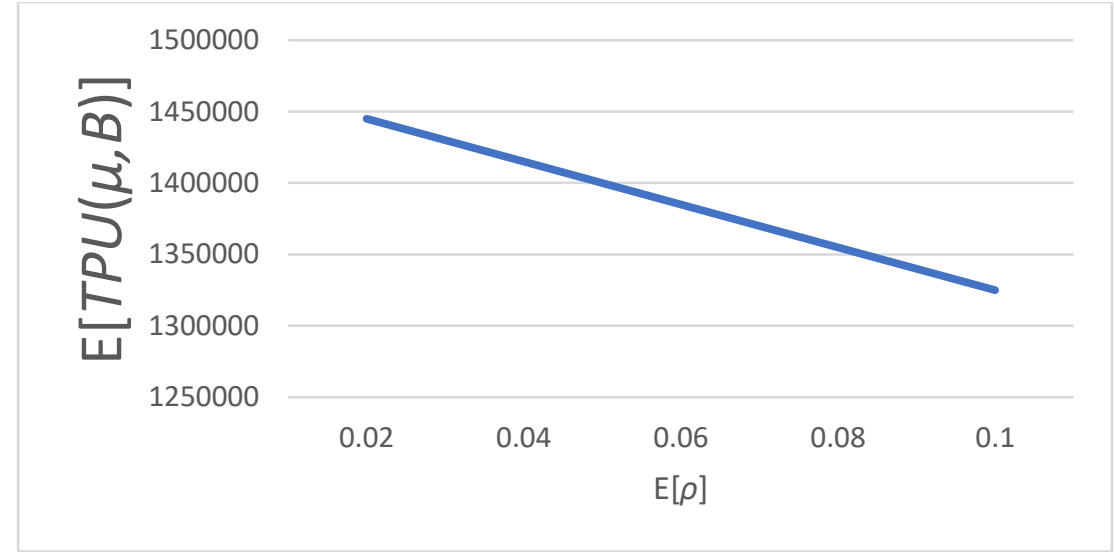

Figure 3. Effect of fraction of defective items $(E[\rho])$ on optimal expected total profit

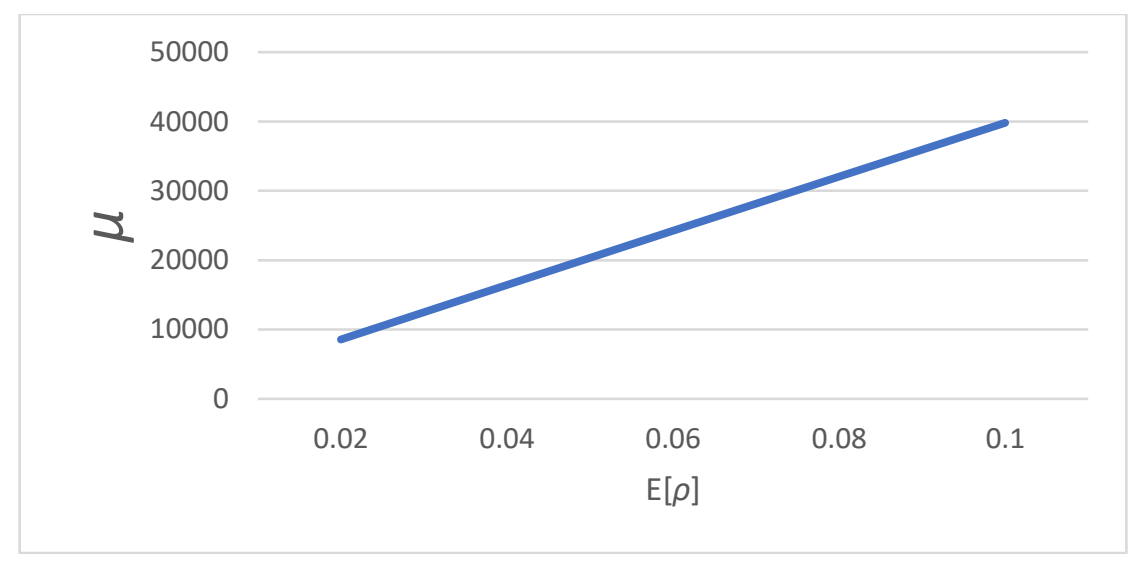

Figure 4. Effect of fraction of defective items $(E[\rho])$ on optimal order quantity $\left(\mu^{*}\right)$

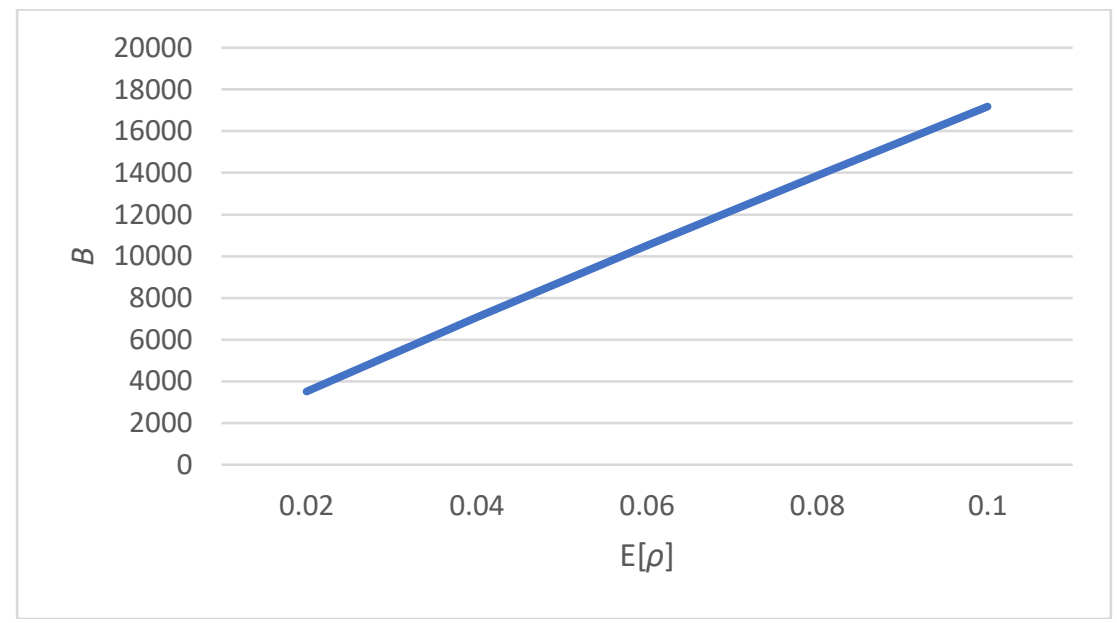

Figure 5. Effect of fraction of defective items $(E[\rho])$ on backorder quantity $\left(B^{*}\right)$ 
International Journal of Mathematical, Engineering and Management Sciences

Vol. 5, No. 5, 869-885, 2020

https://doi.org/10.33889/IJMEMS.2020.5.5.067

Figure 3 reveals that the expected total profit decreases significantly as the fraction of imperfect items increases while the optimal order quantity and backorder quantity increase as the fraction of imperfect items increases (see Figure 4 and Figure 5).

\section{Conclusion}

This research work proposes a two-warehouse inventory model with shortages by seeing the impact of defective items. It was demonstrated as percentage of defective items increases then the expected total profit decreases. This inventory model extends the inventory model of Chung et al. (2009) by including shortages with full backordering. The proposed inventory model shows that expected total profit increases as compared to the expected total profit of Chung et al. (2009)'s inventory model. The proposed inventory can be extended under carbon emission constraints with international supply chain, trade credit and deterioration.

\section{Conflict of Interest}

The authors confirm that there is no conflict of interest to declare for this publication.

\section{Acknowledgements}

The authors would like to express their sincere thanks to the editor and anonymous reviews for their time and valuable suggestions.

\section{References}

Cárdenas-Barrón, L.E. (2000). Observation on: "Economic production quantity model for items with imperfect quality" [Int. J. of Production Economics 64 (2000) 59-64]. International Journal of Production Economics, 67(2), 201-201.

Chung, K.J., Her, C.C., \& Lin, S.D. (2009). A two-warehouse inventory model with imperfect quality production processes. Computers \& Industrial Engineering, 56(1), 193-197.

Das, B., Maity, K., \& Maiti, M. (2007). A two-warehouse supply-chain model under possibility/necessity/credibility measures. Mathematical and Computer Modelling, 46(3-4), 398-409.

Eroglu, A., \& Ozdemir, G. (2007). An economic order quantity model with defective items and shortages. International Journal of Production Economics, 106(2), 544-549.

Goyal, S.K., \& Cárdenas-Barrón, L.E. (2002). Note on: economic production quantity model for items with imperfect quality-a practical approach. International Journal of Production Economics, 77(1), 85-87.

Hsieh, T.P., Dye, C.Y., \& Ouyang, L.Y. (2008). Determining optimal lot size for a two-warehouse system with deterioration and shortages using net present value. European Journal of Operational Research, 191(1), 182-192.

Jaggi, C.K., Cárdenas-Barrón, L.E., Tiwari, S., \& Shafi, A. (2017). Two-warehouse inventory model for deteriorating items with imperfect quality under the conditions of permissible delay in payments. Scientia Iranica, 24(1), 390-412.

Jaggi, C.K., Khanna, A., Pareek, S., \& Sharma, R. (2013). Ordering policy in a two-warehouse environment for deteriorating items with shortages under inflationary conditions. International Journal of Strategic Decision Sciences, 4(2), 27-47. 
International Journal of Mathematical, Engineering and Management Sciences

Vol. 5, No. 5, 869-885, 2020

https://doi.org/10.33889/IJMEMS.2020.5.5.067

Jaggi, C.K., Pareek, S., Khanna, A., \& Sharma, R. (2014). Credit financing in a two-warehouse environment for deteriorating items with price-sensitive demand and fully backlogged shortages. Applied Mathematical Modelling, 38(21-22), 5315-5333.

Jaggi, C.K., Pareek, S., Khanna, A., \& Sharma, R. (2015a). Two-warehouse inventory model for deteriorating items with price-sensitive demand and partially backlogged shortages under inflationary conditions. International Journal of Industrial Engineering Computations, 6(1), 59-80.

Jaggi, C.K., Tiwari, S., \& Shafi, A. (2015b). Effect of deterioration on two-warehouse inventory model with imperfect quality. Computers \& Industrial Engineering, 88, 378-385.

Maddah, B., \& Jaber, M.Y. (2008). Economic order quantity for items with imperfect quality: revisited. International Journal of Production Economics, 112(2), 808-815.

Mukhopadhyay, A., \& Goswami, A. (2017). An inventory model with shortages for imperfect items using substitution of two products. International Journal of Operational Research, 30(2), 193-219.

Papachristos, S., \& Konstantaras, I. (2006). Economic ordering quantity models for items with imperfect quality. International Journal of Production Economics, 100(1), 148-154.

Salameh, M.K., \& Jaber, M.Y. (2000). Economic production quantity model for items with imperfect quality. International Journal of Production Economics, 64(1-3), 59-64.

Sarkar, B. (2012). An inventory model with reliability in an imperfect production process. Applied Mathematics and Computation, 218(9), 4881-4891.

Tiwari, S., Cárdenas-Barrón, L. E., Khanna, A., \& Jaggi, C.K. (2016). Impact of trade credit and inflation on retailer's ordering policies for non-instantaneous deteriorating items in a two-warehouse environment. International Journal of Production Economics, 176, 154-169.

Wee, H.M., Yu, J., \& Chen, M.C. (2007). Optimal inventory model for items with imperfect quality and shortage backordering. Omega, 35(1), 7-11.

Yadav, R., Pareek, S., \& Mittal, M. (2018). Supply chain model for imperfect quality items with trade credit financing: a game theoretical approach. Investigación Operacional, 39(2), 265-277.

Yang, H.L. (2004). Two-warehouse inventory models for deteriorating items with shortages under inflation. European Journal of Operational Research, 157(2), 344-356.

\section{Appendix A.}

Case I. When $t_{1} \geq t_{\omega}$

$$
\begin{aligned}
& \frac{\partial E[T P U(\mu, B)]}{\partial \mu}=\frac{1}{((1-E[\rho]) \mu+B E[\rho])}\left(R \left(s(1-E[\rho])+v E[\rho]-j-d-h_{r}\left(\frac{(\mu-\omega-B)(1-E[\rho])^{2}}{R}+\right.\right.\right. \\
& \left.\left.\left.\frac{2 E[\rho](\mu-\omega-B)}{x}\right)-h_{\omega} \frac{\omega(1-E[\rho])^{2}}{R}\right)\right)-\frac{1}{((1-E[\rho]) \mu+B E[\rho])^{2}}(R(s \mu(1-E[\rho])+v E[\rho] \mu-K-j \mu- \\
& d \mu-h_{r}\left(\frac{1}{2} \frac{(\mu-\omega-B)^{2}(1-E[\rho])^{2}}{R}+\frac{E[\rho](\mu-\omega-B)^{2}}{x}\right)-h_{\omega}\left(\frac{\omega(\mu-\omega-B)(1-E[\rho])^{2}}{R}+\frac{\omega^{2} E[\rho]}{x}+\right. \\
& \left.\left.\left.\frac{\omega^{2}(1-E[\rho])^{2}}{2 R}\right)-\frac{B^{2} b}{2 R}\right)(1-E[\rho])\right)=0
\end{aligned}
$$


International Journal of Mathematical, Engineering and Management Sciences

Vol. 5, No. 5, 869-885, 2020

https://doi.org/10.33889/IJMEMS.2020.5.5.067

$$
\begin{aligned}
& \frac{\partial E[T P U(\mu, B)]}{\partial B}=\frac{1}{((1-E[\rho]) \mu+B E[\rho])}\left(R\left(h_{r}\left(\frac{(\mu-\omega-B)(1-E[\rho])^{2}}{R}+\frac{2 E[\rho](\mu-\omega-B)}{x}\right)\right)+h_{\omega} \frac{\omega(1-E[\rho])^{2}}{R}-\right. \\
& \left.\frac{B b}{R}\right)-\frac{1}{((1-E[\rho]) \mu+B E[\rho])^{2}}(R(s \mu(1-E[\rho])+v E[\rho] \mu-K-j \mu-d \mu- \\
& h_{r}\left(\frac{1}{2} \frac{(\mu-\omega-B)^{2}(1-E[\rho])^{2}}{R}+\frac{E[\rho](\mu-\omega-B)^{2}}{x}\right)-h_{\omega}\left(\frac{\omega(\mu-\omega-B)(1-\mathrm{E}[\rho])^{2}}{R}+\frac{\omega^{2} \mathrm{E}[\rho]}{x}+\frac{\omega^{2}(1-\mathrm{E}[\rho])^{2}}{2 R}\right)- \\
& \left.\left.\frac{B^{2} b}{2 R}\right) E[\rho]\right)=0
\end{aligned}
$$

$\frac{\partial^{2} E[T P U(\mu, B)]}{\partial \mu^{2}}=\frac{-R h_{r}}{((1-E[\rho]) \mu+B E[\rho])}\left(\frac{(1-E[\rho])^{2}}{R}+\frac{2 E[\rho]}{x}\right)-\frac{1}{((1-E[\rho]) \mu+B E[\rho])^{2}}(2 R(s(1-E[\rho])+$ $\left.\left.v E[\rho]-j-d-h_{r}\left(\frac{(\mu-\omega-B)(1-E[\rho])^{2}}{R}+\frac{2 E[\rho](\mu-\omega-B)}{x}\right)-h_{\omega}\left(\frac{\omega(1-\mathrm{E}[\rho])^{2}}{R}\right)\right)(1-E[\rho])\right)+$ $\frac{2 R}{((1-E[\rho]) \mu+B E[\rho])^{3}}\left(s \mu(1-E[\rho])+v E[\rho] \mu-K-j \mu-d \mu-h_{r}\left(\frac{1}{2} \frac{(\mu-\omega-B)^{2}(1-E[\rho])^{2}}{R}+\right.\right.$ $\left.\left.\frac{E[\rho](\mu-\omega-B)^{2}}{x}\right)-h_{\omega}\left(\frac{\omega(\mu-\omega-B)(1-\mathrm{E}[\rho])^{2}}{R}+\frac{\omega^{2} \mathrm{E}[\rho]}{x}+\frac{\omega^{2}(1-\mathrm{E}[\rho])^{2}}{2 R}\right)-\frac{B^{2} b}{2 R}\right)\left(1-E[\rho]^{2}\right)$

$$
\frac{\partial^{2} E[T P U(\mu, B)]}{\partial B^{2}}=\frac{R}{((1-E[\rho]) \mu+B E[\rho])}\left(-h_{r}\left(\frac{(1-E[\rho])^{2}}{R}+\frac{2 E[\rho]}{x}\right)-\frac{b}{R}\right)-
$$

$\frac{2 R}{((1-E[\rho]) \mu+B E[\rho])^{2}}\left(h_{r}\left(\frac{(\mu-\omega-B)(1-E[\rho])^{2}}{R}+\frac{2 E[\rho](\mu-\omega-B)}{x}\right)+\frac{h_{\omega} \omega(1-\mathrm{E}[\rho])^{2}}{R}-\frac{B b}{R}\right) E[\rho]+$ $\frac{2 R}{((1-E[\rho]) \mu+B E[\rho])^{3}}\left(s \mu(1-E[\rho])+v E[\rho] \mu-K-j \mu-d \mu-h_{r}\left(\frac{1}{2} \frac{(\mu-\omega-B)^{2}(1-E[\rho])^{2}}{R}+\right.\right.$ $\left.\left.\frac{E[\rho](\mu-\omega-B)^{2}}{x}\right)-h_{\omega}\left(\frac{\omega(\mu-\omega-B)(1-\mathrm{E}[\rho])^{2}}{R}+\frac{\omega^{2} \mathrm{E}[\rho]}{x}+\frac{\omega^{2}(1-\mathrm{E}[\rho])^{2}}{2 R}\right)-\frac{B^{2} b}{2 R}\right) E[\rho]^{2}$

$\frac{\partial^{2} E[T P U(\mu, B)]}{\partial \mu \partial B}=\frac{\partial^{2} E[T P U(\mu, B)]}{\partial B \partial \mu}=\frac{R h_{r}}{((1-E[\rho]) \mu+B E[\rho])}\left(\frac{(1-E[\rho])^{2}}{R}+\frac{2 E[\rho]}{x}\right)-$

$\frac{R}{((1-E[\rho]) \mu+B E[\rho])^{2}}\left(h_{r}\left(\frac{(\mu-\omega-B)(1-E[\rho])^{2}}{R}+\frac{2 E[\rho](\mu-\omega-B)}{x}\right)+\frac{h_{\omega} \omega(1-\mathrm{E}[\rho])^{2}}{R}-\frac{B b}{R}\right)(1-E[\rho])-$ $\frac{1}{((1-E[\rho]) \mu+B E[\rho])^{2}}\left(R\left(s(1-E[\rho])+v E[\rho]-j-d-h_{r}\left(\frac{(\mu-\omega-B)(1-E[\rho])^{2}}{R}+\frac{2 E[\rho](\mu-\omega-B)}{x}\right)-\right.\right.$ $\left.\left.h_{\omega}\left(\frac{\omega(1-\mathrm{E}[\rho])^{2}}{R}\right)\right) E[\rho]\right)+\frac{2 R}{((1-E[\rho]) \mu+B E[\rho])^{3}}(s \mu(1-E[\rho])+v E[\rho] \mu-K-j \mu-d \mu-$ $h_{r}\left(\frac{1}{2} \frac{(\mu-\omega-B)^{2}(1-E[\rho])^{2}}{R}+\frac{E[\rho](\mu-\omega-B)^{2}}{x}\right)-h_{\omega}\left(\frac{\omega(\mu-\omega-B)(1-\mathrm{E}[\rho])^{2}}{R}+\frac{\omega^{2} \mathrm{E}[\rho]}{x}+\frac{\omega^{2}(1-\mathrm{E}[\rho])^{2}}{2 R}\right)-$ $\left.\frac{B^{2} b}{2 R}\right)(1-E[\rho]) E[\rho]$ 
International Journal of Mathematical, Engineering and Management Sciences

Vol. 5, No. 5, 869-885, 2020

https://doi.org/10.33889/IJMEMS.2020.5.5.067

Appendix B. Case II. When $t_{1}<t_{\omega}$.

$$
\begin{aligned}
& \frac{\partial E[T P U(\mu, B)]}{\partial \mu}=\frac{1}{((1-E[\rho]) \mu+B E[\rho])}\left(R \left(s(1-E[\rho])+v E[\rho]-j-d-h_{r}\left(\frac{(\mu-\omega-B)(1-E[\rho])^{2}}{R}+\right.\right.\right. \\
& \left.\frac{2 E[\rho](\mu-\omega-B)}{x}\right)-h_{\omega}\left(\frac{\omega(1-E[\rho])}{R}-\left(\frac{\omega}{x}-\frac{(\mu-\omega-B)(1-E[\rho])}{R}\right)(1-E[\rho])-\right. \\
& \frac{(1-E[\rho])\left(\omega-R\left(\frac{\omega}{x}-\frac{(\mu-\omega-B)(1-E[\rho])}{R}\right)\right)}{R}+\frac{1}{2} \frac{(1-E[\rho])\left(\omega-R\left(\frac{\omega}{x}-\frac{(\mu-\omega-B)(1-E[\rho])}{R}\right)-E[\rho] \omega\right)}{R}+ \\
& \left.\left.\frac{1}{2}\left(\frac{(\mu-\omega-B)(1-E[\rho])+\omega(1-E[\rho])}{R}-\frac{\omega}{x}\right)(1-E[\rho])\right)\right)-\frac{1}{((1-E[\rho]) \mu+B E[\rho])^{2}}(R(s \mu(1-E[\rho])+
\end{aligned}
$$$$
v E[\rho] \mu-K-j \mu-d \mu-h_{r}\left(\frac{1}{2} \frac{(\mu-\omega-B)^{2}(1-E[\rho])^{2}}{R}+\frac{E[\rho](\mu-\omega-B)^{2}}{x}\right)-h_{\omega}\left(\frac{\omega(\mu-\omega-B)(1-E[\rho])}{R}+\right.
$$$$
R\left(\frac{\omega}{x}-\frac{(\mu-\omega-B)(1-E[\rho])}{R}\right)^{2}+\left(\frac{\omega}{x}-\frac{(\mu-\omega-B)(1-E[\rho])}{R}\right)\left(\omega-R\left(\frac{\omega}{x}-\frac{(\mu-\omega-B)(1-E[\rho])}{R}\right)\right)+
$$$$
\left.\left.\frac{1}{2}\left(\frac{(\mu-\omega-B)(1-E[\rho])+\omega(1-E[\rho])}{R}-\frac{\omega}{x}\right)\left(\omega-R\left(\frac{\omega}{x}-\frac{(\mu-\omega-B)(1-E[\rho])}{R}\right)-E[\rho] \omega\right)\right)+\frac{B^{2} b}{2 R}\right)(1-
$$$$
E[\rho]))=0
$$

$$
\begin{aligned}
& \frac{\partial E[T P U(\mu, B)]}{\partial B}=\frac{1}{(1-E[\rho]) \mu+B E[\rho]}\left(R \left(h_{r}\left(\frac{(\mu-\omega-B)(1-E[\rho])^{2}}{R}+\frac{2 E[\rho](\mu-\omega-B)}{x}\right)-h_{\omega}\left(\frac{-\omega(1-E[\rho])}{R}+\right.\right.\right. \\
& 2\left(\frac{\omega}{x}-\frac{(\mu-\omega-B)(1-E[\rho])}{R}\right)(1-E[\rho])+\frac{(1-E[\rho])\left(\omega-R\left(\frac{\omega}{x}-\frac{(\mu-\omega-B)(1-E[\rho])}{R}\right)\right)}{R}+\left(\frac{\omega}{x}-\right. \\
& \left.\frac{(\mu-\omega-B)(1-E[\rho])}{R}\right)(-1+E[\rho])+\frac{(-1+E[\rho])\left(\omega-R\left(\frac{\omega}{x}-\frac{(\mu-\omega-B)(1-E[\rho])}{R}\right)\right)-E[\rho] \omega}{2 R}+
\end{aligned}
$$


International Journal of Mathematical, Engineering and Management Sciences

Vol. 5, No. 5, 869-885, 2020

https://doi.org/10.33889/IJMEMS.2020.5.5.067

$\left.\left.\left.\frac{1}{2}\left(\frac{(\mu-\omega-B)(1-E[\rho])+\omega(1-E[\rho])}{R}-\frac{\omega}{x}\right)(-1+E[\rho])\right)-\frac{B b}{R}\right)\right)-\frac{1}{((1-E[\rho]) \mu+B E[\rho])^{2}}(R(s \mu(1-$
$E[\rho])+v E[\rho] \mu-K-j \mu-d \mu-h_{r}\left(\frac{1}{2} \frac{(\mu-\omega-B)^{2}(1-E[\rho])^{2}}{R}+\frac{E[\rho](\mu-\omega-B)^{2}}{x}\right)-$
$h_{\omega}\left(\frac{\omega(\mu-\omega-B)(1-E[\rho])}{R}+R\left(\frac{\omega}{x}-\frac{(\mu-\omega-B)(1-E[\rho])}{R}\right)^{2}+\left(\frac{\omega}{x}-\frac{(\mu-\omega-B)(1-E[\rho])}{R}\right)(\omega-\right.$
$\left.R\left(\frac{\omega}{x}-\frac{(\mu-\omega-B)(1-E[\rho])}{R}\right)\right)+\frac{1}{2}\left(\frac{(\mu-\omega-B)(1-E[\rho])+\omega(1-E[\rho])}{R}-\frac{\omega}{x}\right)\left(\omega-R\left(\frac{\omega}{x}-\frac{(\mu-\omega-B)(1-E[\rho])}{R}\right)-\right.$
$\left.\left.E[\rho] \omega))+\frac{B^{2} b}{2 R}\right) E[\rho]\right)=0$

$\frac{\partial^{2} E[T P U(\mu, B)]}{\partial \mu^{2}}=\frac{R}{(1-E[\rho]) \mu+B E[\rho]}\left(-h_{r}\left(\frac{(1-E[\rho])^{2}}{R}+\frac{2 E[\rho]}{x}\right)-h_{\omega}\left(\frac{(1-E[\rho])^{2}}{R}\right)\right)-$

$\frac{1}{((1-E[\rho]) \mu+B E[\rho])^{2}}\left(2 R\left(s(1-E[\rho])+v E[\rho]-j-d-h_{r}\left(\frac{(\mu-\omega-B)(1-E[\rho])^{2}}{R}+\right.\right.\right.$

$\left.\frac{2 E[\rho](\mu-\omega-B)}{x}\right)-h_{\omega}\left(\frac{\omega(1-E[\rho])}{R}-\left(\frac{\omega}{x}-\frac{(\mu-\omega-B)(1-E[\rho])}{R}\right)(1-E[\rho])-\right.$

$\frac{(1-E[\rho])\left(\omega-R\left(\frac{\omega}{x}-\frac{(\mu-\omega-B)(1-E[\rho])}{R}\right)\right)}{R}+\frac{1}{2} \frac{(1-E[\rho])\left(\omega-R\left(\frac{\omega}{x}-\frac{(\mu-\omega-B)(1-E[\rho])}{R}\right)-E[\rho] \omega\right)}{R}+$

$\left.\left.\frac{1}{2}\left(\frac{(\mu-\omega-B)(1-E[\rho])+\omega(1-E[\rho])}{R}-\frac{\omega}{x}\right)(1-E[\rho])\right)(1-E[\rho])\right)+$

$\frac{1}{((1-E[\rho]) \mu+B E[\rho])^{3}}\left(2 R\left(s \mu(1-E[\rho])+v E[\rho] \mu-K-j \mu-d \mu-h_{r}\left(\frac{1}{2} \frac{(\mu-\omega-B)^{2}(1-E[\rho])^{2}}{R}+\right.\right.\right.$

$\left.\frac{E[\rho](\mu-\omega-B)^{2}}{x}\right)-h_{\omega}\left(\frac{\omega(\mu-\omega-B)(1-E[\rho])}{R}+R\left(\frac{\omega}{x}-\frac{(\mu-\omega-B)(1-E[\rho])}{R}\right)^{2}+\left(\frac{\omega}{x}-\right.\right.$

$\left.\frac{(\mu-\omega-B)(1-E[\rho])}{R}\right)\left(\omega-R\left(\frac{\omega}{x}-\frac{(\mu-\omega-B)(1-E[\rho])}{R}\right)\right)+\frac{1}{2}\left(\frac{(\mu-\omega-B)(1-E[\rho])+\omega(1-E[\rho])}{R}-\frac{\omega}{x}\right)(\omega-$ 
International Journal of Mathematical, Engineering and Management Sciences

Vol. 5, No. 5, 869-885, 2020

https://doi.org/10.33889/IJMEMS.2020.5.5.067

$\left.\left.\left.\left.R\left(\frac{\omega}{x}-\frac{(\mu-\omega-B)(1-E[\rho])}{R}\right)-E[\rho] \omega\right)\right)-\frac{B^{2} b}{2 R}\right)\left(1-E[\rho]^{2}\right)\right)$

$\frac{\partial^{2} E[T P U(\mu, B)]}{\partial B^{2}}=\frac{R}{(1-E[\rho]) \mu+B E[\rho]}\left(-h_{r}\left(\frac{(1-E[\rho])^{2}}{R}+\frac{2 E[\rho]}{x}\right)-h_{\omega}\left(\frac{2(1-E[\rho])^{2}}{R}+\frac{2(1-E[\rho])(-1+E[\rho])}{R}+\right.\right.$ $\left.\left.\frac{(-1+E[\rho])^{2}}{R}\right)-\frac{b}{R}\right)-\frac{2 R}{((1-E[\rho]) \mu+B E[\rho])^{2}}\left(h_{r}\left(\frac{(\mu-\omega-B)(1-E[\rho])^{2}}{R}+\frac{2 E[\rho](\mu-\omega-B)}{x}\right)-\right.$

$h_{\omega}\left(\frac{-\omega(1-E[\rho])}{R}+2\left(\frac{\omega}{x}-\frac{(\mu-\omega-B)(1-E[\rho])}{R}\right)(1-E[\rho])+\frac{(1-E[\rho])\left(\omega-R\left(\frac{\omega}{x}-\frac{(\mu-\omega-B)(1-E[\rho])}{R}\right)\right)}{R}+\right.$ $\left(\frac{\omega}{x}-\frac{(\mu-\omega-B)(1-E[\rho])}{R}\right)(-1+E[\rho])+\frac{(-1+E[\rho])\left(\omega-R\left(\frac{\omega}{x}-\frac{(\mu-\omega-B)(1-E[\rho])}{R}\right)\right)-E[\rho] \omega}{2 R}+$ $\left.\left.\frac{1}{2}\left(\frac{(\mu-\omega-B)(1-E[\rho])+\omega(1-E[\rho])}{R}-\frac{\omega}{x}\right)(-1+E[\rho])\right)-\frac{B b}{R}\right) E[\rho]+\frac{1}{((1-E[\rho]) \mu+B E[\rho])^{3}}(2 R(s \mu(1-$ $E[\rho])+v E[\rho] \mu-K-j \mu-d \mu-h_{r}\left(\frac{1}{2} \frac{(\mu-\omega-B)^{2}(1-E[\rho])^{2}}{R}+\frac{E[\rho](\mu-\omega-B)^{2}}{x}\right)-$ $h_{\omega}\left(\frac{\omega(\mu-\omega-B)(1-E[\rho])}{R}+R\left(\frac{\omega}{x}-\frac{(\mu-\omega-B)(1-E[\rho])}{R}\right)^{2}+\left(\frac{\omega}{x}-\frac{(\mu-\omega-B)(1-E[\rho])}{R}\right)(\omega-\right.$ $\left.R\left(\frac{\omega}{x}-\frac{(\mu-\omega-B)(1-E[\rho])}{R}\right)\right)+\frac{1}{2}\left(\frac{(\mu-\omega-B)(1-E[\rho])+\omega(1-E[\rho])}{R}-\frac{\omega}{x}\right)\left(\omega-R\left(\frac{\omega}{x}-\frac{(\mu-\omega-B)(1-E[\rho])}{R}\right)-\right.$ $\left.\left.E[\rho] \omega))-\frac{B^{2} b}{2 R}\right) E[\rho]^{2}\right)$

$\frac{\partial^{2} E[T P U(\mu, B)]}{\partial \mu \partial B}=\frac{\partial^{2} E[T P U(\mu, B)]}{\partial B \partial \mu}=\frac{R}{(1-E[\rho]) \mu+B E[\rho]}\left(h_{r}\left(\frac{(1-E[\rho])^{2}}{R}+\frac{2 E[\rho]}{x}\right)+h_{\omega}\left(\frac{(1-E[\rho])^{2}}{R}\right)\right)-$ $\frac{1}{((1-E[\rho]) \mu+B E[\rho])^{2}}\left(R\left(h_{r}\left(\frac{(\mu-\omega-B)(1-E[\rho])^{2}}{R}+\frac{2 E[\rho](\mu-\omega-B)}{x}\right)-h_{\omega}\left(\frac{-\omega(1-E[\rho])}{R}+\right.\right.\right.$ $2\left(\frac{\omega}{x}-\frac{(\mu-\omega-B)(1-E[\rho])}{R}\right)(1-E[\rho])+\frac{(1-E[\rho])\left(\omega-R\left(\frac{\omega}{x}-\frac{(\mu-\omega-B)(1-E[\rho])}{R}\right)\right)}{R}+\left(\frac{\omega}{x}-\right.$ 
International Journal of Mathematical, Engineering and Management Sciences

Vol. 5, No. 5, 869-885, 2020

https://doi.org/10.33889/IJMEMS.2020.5.5.067

$$
\begin{aligned}
& \left.\frac{(\mu-\omega-B)(1-E[\rho])}{R}\right)(-1+E[\rho])+\frac{(-1+E[\rho])\left(\omega-R\left(\frac{\omega}{x}-\frac{(\mu-\omega-B)(1-E[\rho])}{R}\right)\right)-E[\rho] \omega}{2 R}+ \\
& \left.\left.\left.\frac{1}{2}\left(\frac{(\mu-\omega-B)(1-E[\rho])+\omega(1-E[\rho])}{R}-\frac{\omega}{x}\right)(-1+E[\rho])\right)-\frac{B b}{R}\right)(1-E[\rho])\right)- \\
& \frac{1}{((1-E[\rho]) \mu+B E[\rho])^{2}}\left(R \left(s(1-E[\rho])+v E[\rho]-j-d-h_{r}\left(\frac{(\mu-\omega-B)(1-E[\rho])^{2}}{R}+\frac{2 E[\rho](\mu-\omega-B)}{x}\right)-\right.\right. \\
& h_{\omega}\left(\frac{\omega(1-E[\rho])}{R}-\left(\frac{\omega}{x}-\frac{(\mu-\omega-B)(1-E[\rho])}{R}\right)(1-E[\rho])-\frac{(1-E[\rho])\left(\omega-R\left(\frac{\omega}{x}-\frac{(\mu-\omega-B)(1-E[\rho])}{R}\right)\right)}{R}+\right. \\
& \frac{1}{2} \frac{(1-E[\rho])\left(\omega-R\left(\frac{\omega}{x}-\frac{(\mu-\omega-B)(1-E[\rho])}{R}\right)-E[\rho] \omega\right)}{R}+\frac{1}{2}\left(\frac{(\mu-\omega-B)(1-E[\rho])+\omega(1-E[\rho])}{R}-\frac{\omega}{x}\right)(1- \\
& E[\rho])) E[\rho])+\frac{1}{((1-E[\rho]) \mu+B E[\rho])^{3}}(2 R(s \mu(1-E[\rho])+v E[\rho] \mu-K-j \mu-d \mu- \\
& h_{r}\left(\frac{1}{2} \frac{(\mu-\omega-B)^{2}(1-E[\rho])^{2}}{R}+\frac{E[\rho](\mu-\omega-B)^{2}}{x}\right)-h_{\omega}\left(\frac{\omega(\mu-\omega-B)(1-E[\rho])}{R}+R\left(\frac{\omega}{x}-\frac{(\mu-\omega-B)(1-E[\rho])}{R}\right)^{2}+\right. \\
& \left(\frac{\omega}{x}-\frac{(\mu-\omega-B)(1-E[\rho])}{R}\right)\left(\omega-R\left(\frac{\omega}{x}-\frac{(\mu-\omega-B)(1-E[\rho])}{R}\right)\right)+\frac{1}{2}\left(\frac{(\mu-\omega-B)(1-E[\rho])+\omega(1-E[\rho])}{R}-\right. \\
& \left.\left.\left.\left.\frac{\omega}{x}\right)\left(\omega-R\left(\frac{\omega}{x}-\frac{(\mu-\omega-B)(1-E[\rho])}{R}\right)-E[\rho] \omega\right)\right)-\frac{B^{2} b}{2 R}\right)(1-E[\rho]) E[\rho]\right)
\end{aligned}
$$

